\title{
Los superhéroes como herramienta para el aprendizaje: la ciencia ficción llega a las aulas
}

Ramón Fernández, Francisca ${ }^{a}$, Bosch Roig, Pilar ${ }^{\mathrm{b}}$, Cabedo Mallol, Vicente ${ }^{\mathrm{c}}$, Casar Furió, María Emilia $^{\mathrm{d}}$, Giménez Chornet, Vicente ${ }^{\mathrm{e}}$, Hernández Guijarro, Fernando ${ }^{\mathrm{f}}$, Lull Noguera, Cristina ${ }^{\mathrm{g}}$, Oltra Gutiérrez, Juan Vicente $^{\text {h }}$, Osete Cortina, Laura ${ }^{i}$, Soriano Soto, María Desamparados ${ }^{j}$

aProfesora titular. Departamento de Urbanismo, Universitat Politècnica de València, frarafer@urb.upv.es, ${ }^{\text {b }}$ Profesora asociada. Departamento de Conservación y Restauración de Bienes Culturales, Universitat Politècnica de València, mabosroi@upvnet.upv.es, 'Profesor titular. Departamento de Urbanismo, Universitat Politècnica de València, vicamal@urb.upv.es; d Profesora titular. Departamento de Urbanismo, Universitat Politècnica de València, macafu@urb.upv.es; ${ }^{\text {e }}$ Profesor titular. Departamento de Comunicación Audiovisual, Documentación e Historia del Arte, Universitat Politècnica de València, vigicho@har.upv.es; ${ }^{f}$ Profesor Ayudante Doctor. Departamento de Economía y Ciencias Sociales, Universitat Politècnica de València, fehergu@upv.es; ${ }^{g}$ Profesora Contratada Doctora, Departamento de Química, Universitat Politècnica de València, clull@upvnet.upv.es, ${ }^{\text {h }}$ Profesor titular. Departamento de Organización de Empresas, Universitat Politècnica de València, jvoltra@omp.upv.es; ${ }^{i}$ Profesora asociada, Departamento de Conservación y Restauración de Bienes Culturales, Universitat Politècnica de València, losete@,crbc@upv.es;; ${ }^{j}$ Catedrática. Departamento de Producción Vegetal, Universitat Politècnica de València, asoriano@prv.upv.es

\section{\$EWWDW}

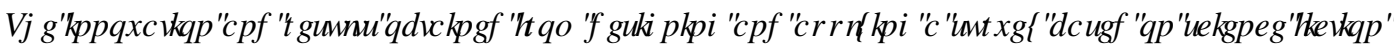

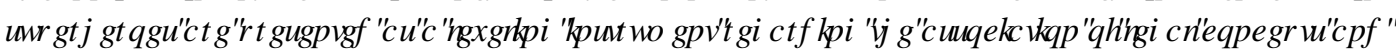

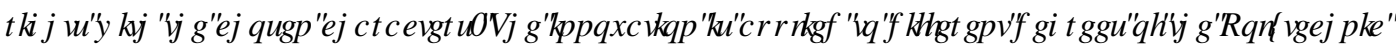

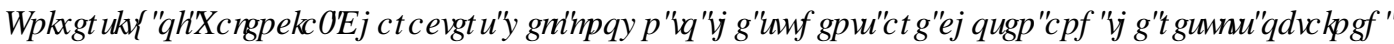

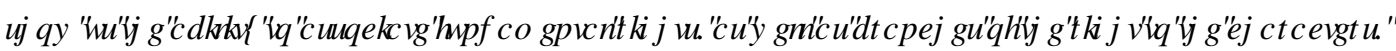

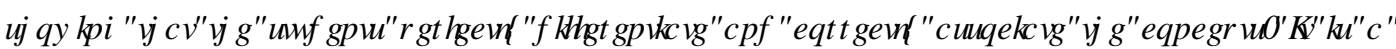

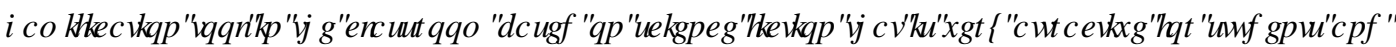

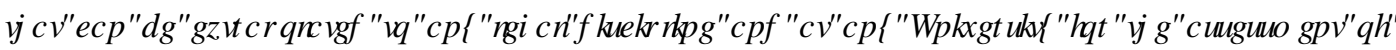
NQRZ QHGJ HEI IWXGHWD

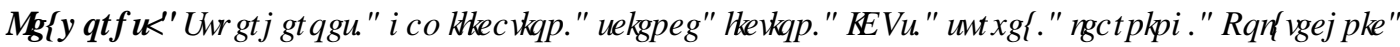

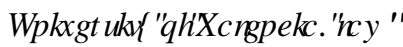

\section{HXP HQ}

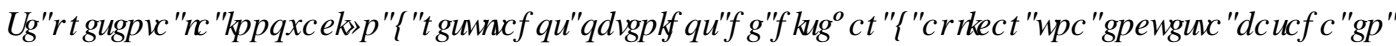

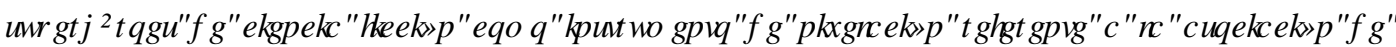

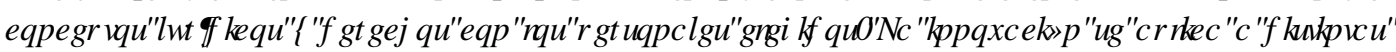

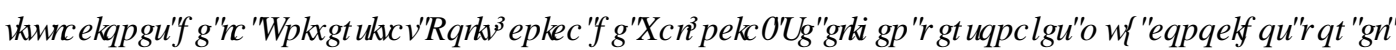

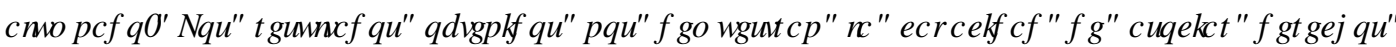

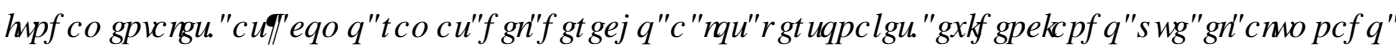

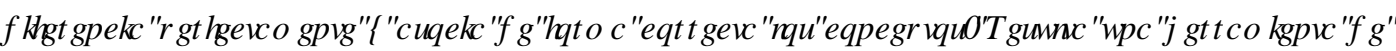

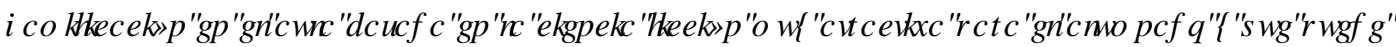

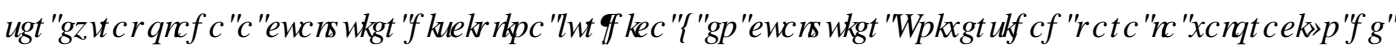
FRQRFIP IHQURVSRLUSDUATGHODOP QDORW

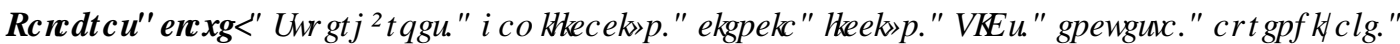

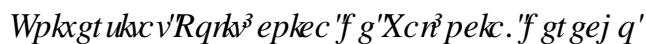




\section{Introducción}

El grupo de innovación docente (EICE), «Recursos Tecnológicos para el aprendizaje jurídico, la documentación y comunicación audiovisual (RETAJUDOCA)» tiene como principal finalidad la utilización de los recursos tecnológicos para el aprendizaje, de forma que se potencien al máximo las tecnologías de la información y comunicación (TICs), aplicándose a la docencia, tanto presencial como a distancia, persiguiendo la finalidad de lograr los objetivos de una máxima participación del alumno y su utilización por parte del docente, como innovación educativa.

Otro aspecto a tener en cuenta es la correcta utilización de las TICs, para proteger y hacer un uso correcto de los recursos evitando una infracción en el ámbito de la propiedad intelectual. Por todo ello, los motivos de constitución de este grupo son la puesta en común de un grupo de profesores del ámbito del Derecho, Documentación, Organización de Empresas, Conservación y Restauración, Economía y Ciencias Sociales, Producción Vegetal y Química que imparten docencia en titulaciones no jurídicas e ingenierías y realizan una adaptación de dichas disciplinas en una Universidad con perfil técnico. Destaca el interés del grupo de profesorado por la innovación docente, muchos de ellos habiendo elaborado material audiovisual específico para complemento de sus asignaturas (objetos de aprendizaje, módulos de aprendizaje y de asignaturas OCV), así como la puesta en común de las nuevas metodologías relacionas con las tecnologías con el objetivo de conseguir la mejora y calidad docente.

Sin embargo, el grupo RETAJUDOCA, como grupo consolidado, quiere ir "más allá", dar un "salto" en su trayectoria y atreverse con diseñar distintas actividades con audiovisuales teniendo como eje temático la ciencia ficción. Las razones por las cuales quiere ampliar su espectro, es precisamente la situación vivida, la docencia online, en un contexto que realmente era de "ciencia ficción", como es una pandemia mundial.

La relación de las disciplinas con el séptimo arte es referenciada por la doctrina especializada (Herrero, 2002; García y Brox, 2010; Martínez de Pisón, 2017; García, 2019), ya que el cine en el ámbito docente ha tenido una gran repercusión, no sólo a través de análisis de películas, sino como recursos para grupos de trabajo en entornos multidisciplinares (Oltra et al., 2016; Ramón, 2019; Ramón et al. 2019a, 2019b y 2019c; Ramón, Lull y Soriano, 2019). Ello se plasma especialmente en las disciplinas jurídicas, en las que la relación Derecho y ciencia ficción ha sido tomada como referencia para el aprendizaje activo (Alerm y González, 2019).

Por ello, una de las últimas innovaciones que estamos llevando a cabo en el marco de la actividad de innovación docente es la gamificación relacionada con la ciencia ficción. Bajo esta premisa, se ha considerado de interés diseñar una encuesta relacionada con superhéroes muy conocidos por el alumnado para fomentar el aprendizaje de conceptos principalmente de carácter jurídico, mediante el sistema de asociación de ideas o conceptos a los personajes. Representa, pues, un ejemplo de gamificación con la finalidad de dinamizar el aprendizaje a través del juego (Prensky, 2002; Pérez-López y Rivera, 2017; Corchuelo, 2018; Moreno, Perales e Hidalgo, 2019; Prieto, 2020).

Esta encuesta de superhéroes de ciencia ficción nos va a servir como nivelación para comprobar el cuál es el conocimiento de aspectos jurídicos mediante la asociación del derecho con los personajes de cómics y de películas, en los que está muy clara la diferencia entre el bien y el mal, el héroe y el villano, y también el antihéroe.

También nos resulta de utilidad para que después el alumnado pueda realizar los casos prácticos jurídicos basados en animación, ficción, documentales, humor y ciencia ficción, ya que le ayudará a contextualizar las situaciones que observe en las series y películas (Ramón et al., 2016, 2017, 2018, 2019, 2021).

La encuesta se pasa en un total de seis asignaturas: 
a) Legislación del patrimonio cultural y creación de pequeñas empresas. Máster Universitario en Conservación y Restauración de Bienes Culturales. 21 alumnos.

b) Aspectos legales y sociológicos de la biotecnología. Grado de Biotecnología. 97 alumnos.

c) Derecho alimentario y protección del consumidor. Grado en Ciencia y Tecnología de Alimento. 35 alumnos.

d) Derecho civil II. Máster en Gestión Administrativa. 19 alumnos.

e) Derecho Espacial. Máster Universitario en Ingeniería Aeronáutica. 4 alumnos.

f) Gobernanza forestal y del Medio Natural. Grado en Ingeniería Forestal y del Medio Natural. 26 alumnos.

Se expondrán los resultados obtenidos en la experiencia, tras el diseño y activación de la encuesta relativa a los superhéroes, que se ha facilitado al alumnado de diversas titulaciones de la Universitat Politècnica de València, con la finalidad de observar su comprensión y asociación del derecho con los personajes de ciencia ficción. Se diseña una encuesta de preguntas abiertas, con lo que permite al alumnado no sólo relacionarlo con el Derecho, sino, incluso, aportar los preceptos jurídicos que considera relacionados con el personaje de ciencia ficción.

\section{Objetivos}

Los principales objetivos son los siguientes:

a) Utilizar una encuesta diseñada de fácil respuesta y con un diseño atractivo, con inclusion de imágenes de superhéroes de ciencia ficción, teniendo como base el empleo de recursos visuales en la línea de los PIMEs que desarrolla el grupo, y en el que está trabajando actualmente basado en la ciencia ficción en entornos presenciales, híbridos y virtuales basados en la gamificación.

b) Incrementar la participación y dinamización en el aula del alumnado de titulación no jurídica, que tiene que conocer conceptos jurídicos y las ramas del derecho de una forma divertida y fácil, al ser personajes muy conocidos, y que establecen de forma clara la diferenciación entre el bien y el mal, así como la situación de colectivos vulnerables.

c) Comprobar el grado de asociación correcta de ramas del derecho (civil, tributario, penal, constitucional, procesal), y de conceptos jurídicos (discriminación, derechos fundamentales, derechos humanos, inmigración, enfermedades que afectan a la capacidad de obrar).

c) Establecer el grado de aceptación de esta innovación basada en la gamificación relacionando la ciencia ficción con personajes de superhéroes para poderlo extrapolar a otro tipo de actividades, por ejemplo, en vez de encuesta abierta, una encuesta cerrada, y asociación con conceptos ya proporcionados.

\section{Diseño de la innovación: selección de los superhéroes y diseño de la encuesta}

\subsection{Personajes incluidos y formato elegido}

Se diseña una encuesta en formato forms de office, para que a través de un enlace proporcionado por el profesorado, el alumnado conteste de forma cómoda y fácil a la encuesta.

Se establece una encuesta breve, de 11 cuestiones, y de respuestas abiertas. La encuesta se titula "Derecho y personajes de ciencia ficción” y la pregunta única para cada personaje es la siguiente: “¿Con qué derecho relacionarías cada personaje?" (Fig. 1). 


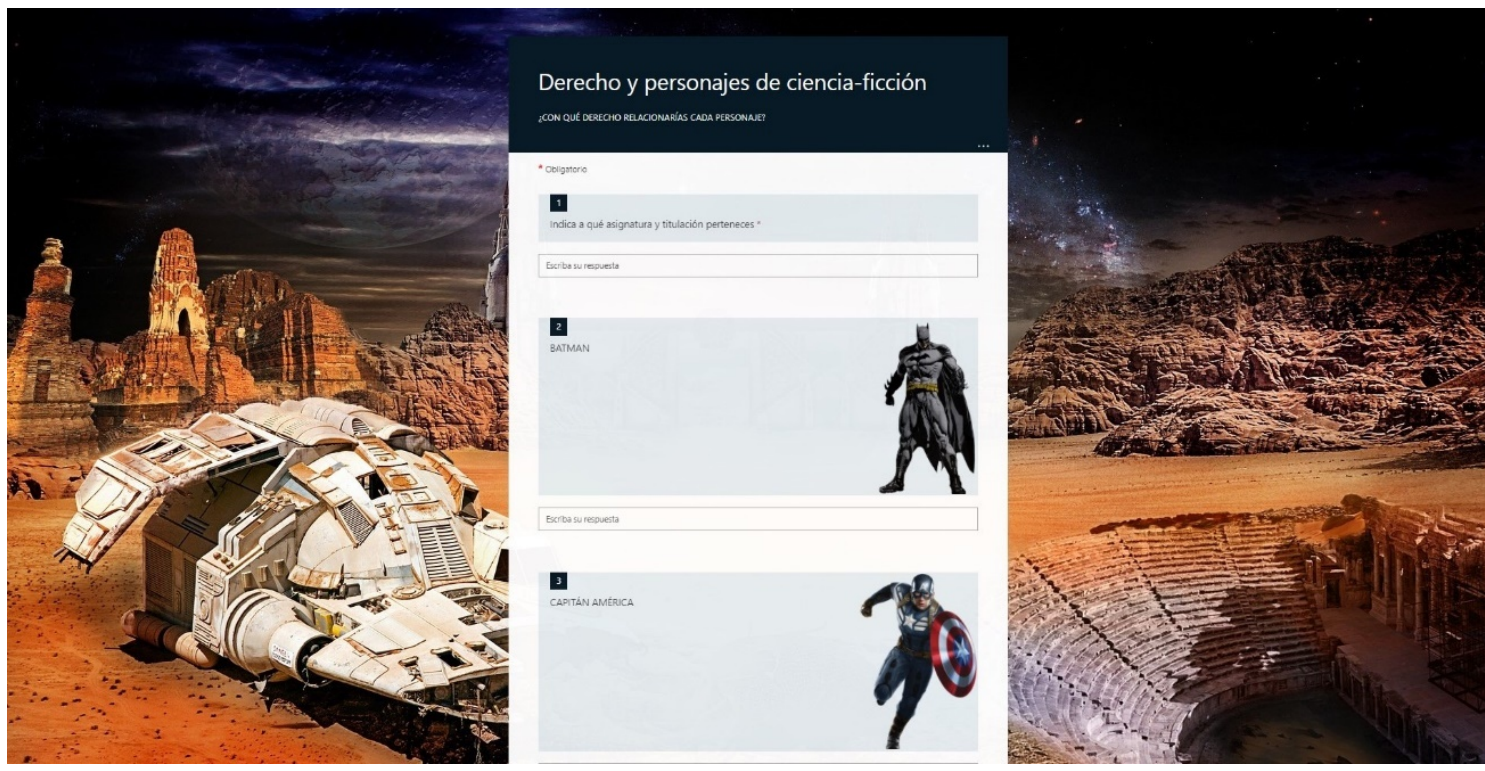

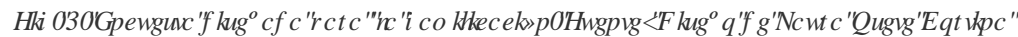

Para la elección de los superhéroes se tuvieron en cuenta que fueran personajes de ciencia fícción tanto clásicos como más modernos, pero que resultaran muy conocidos para el alumnado y que mostrara aspectos muy claros de diferenciación entre el bien y el mal, así como que fueran vulnerables.

Se eligen los siguientes personajes, incluyendo una imagen de los mismos, para su mejor identificación por parte del alumnado. Todos estos personajes son protagonistas de series y películas de ciencia fícción que han tenido mucho éxito y que establecen de forma muy clara la dualidad entre el bien y el mal, así como el héroe y villano, y también el antihéroe. En todos existe una relación con el ámbito jurídico, bien a través de la figura del redentor, y principalmente de la justicia en la sociedad. También se han incluido personajes marginados y vulnerables, así como los que padecen alguna afección psiquiátrica, con la finalidad de observar la identificación por parte del alumnado.

Se eligen un total de 10 personajes, en el que destacan que son todos masculinos, ya que en el ámbito de la ciencia ficción no resulta fácil encontrar personajes femeninos (Fig. 2).

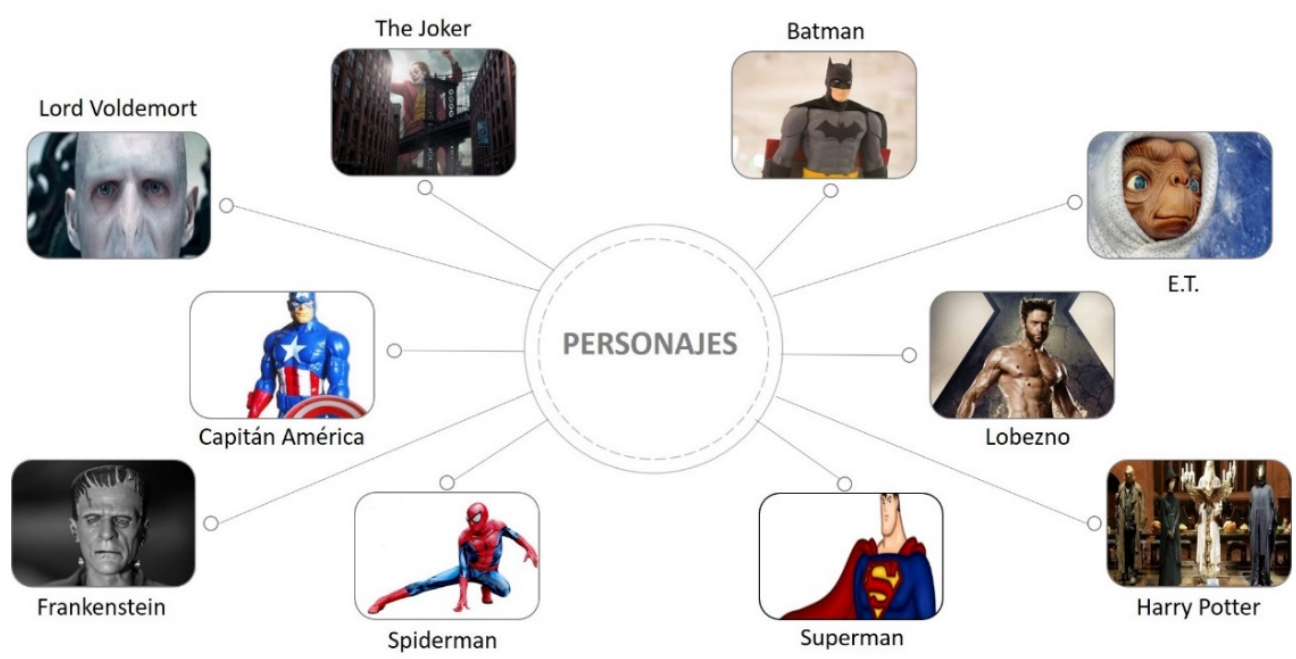

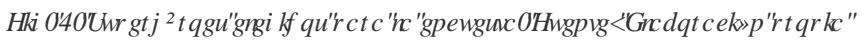

[

(c)) BY-NC-ND 2021, Universitat Politècnica de València

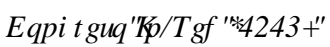




\subsection{Encuesta de respuestas abiertas}

Se elige la opción de que el alumnado conteste de forma abierta, con la finalidad de no influirle en un listado predeterminado de conceptos, y comprobar cuál es su grado de asociación.

En un recuadro al final de la figura del superhéroe, dispone de una casilla para escribir su opinión (Fig. 3).
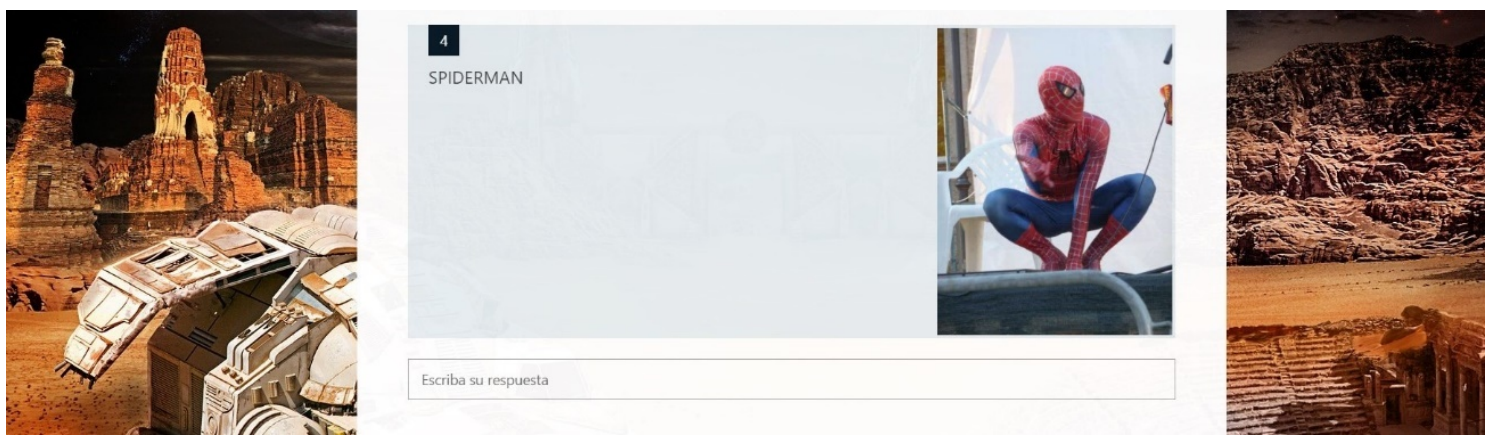

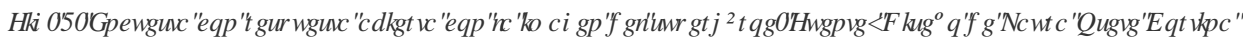

\section{Desarrollo de la innovación: realización de la encuesta y su aplicación a distintas asignaturas y titulaciones de la Universitat Politènica de València}

Se realizó el pase de la encuesta de superhéroes a distintas titulaciones de la Universitat Politècnica de València., que hemos referido anteriormente.

Interesa destacar que la encuesta tenía la pretensión de valorar el grado de conocimiento y asociación del derecho, ramas jurídicas y conceptos en relación con los superhéroes.

La encuesta se pasó tanto en asignaturas del primer cuatrimestre como del segundo, y se obtienen un total de 21 respuestas por parte del alumnado.

La encuestas es totalmente online, con adaptación al horario del alumnado, y con la posibilidad de realizarla solamente una vez por alumno.

\section{Resultados}

Como hemos indicado se obtuvieron 21 respuestas en total en las que hemos podido comprobar el alto grado de asociación de los conceptos en relación con los superhéroes y el derecho. 
Vamos a ver los resultados por personajes:

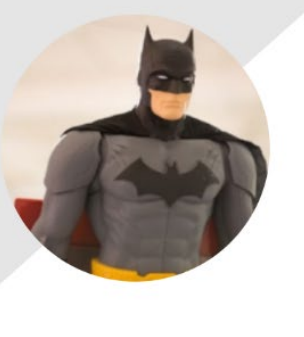

\section{Batman}
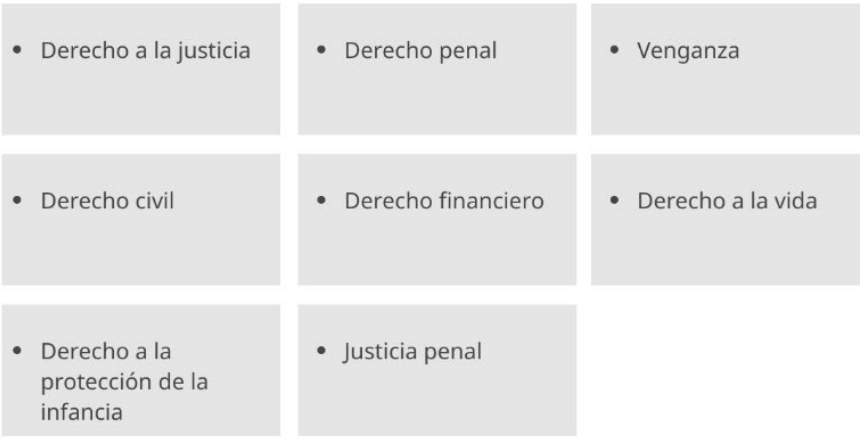

- Justicia

- Derecho a la vida

- Derecho a la

dignidad humana y

a la intimidad

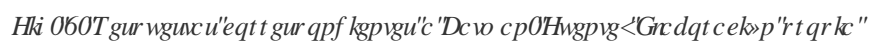

\section{Capitán América}
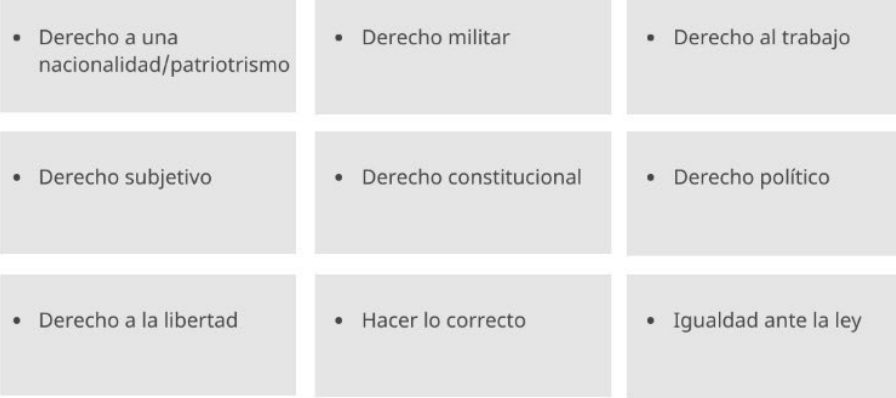

- Justicia

- Derecho romano

- Principio de legalidad penal

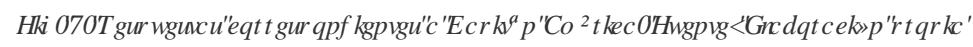




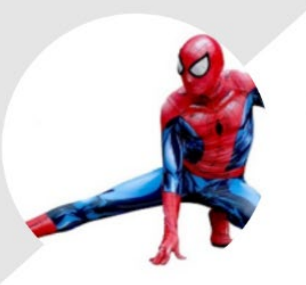

\section{Spiderman}
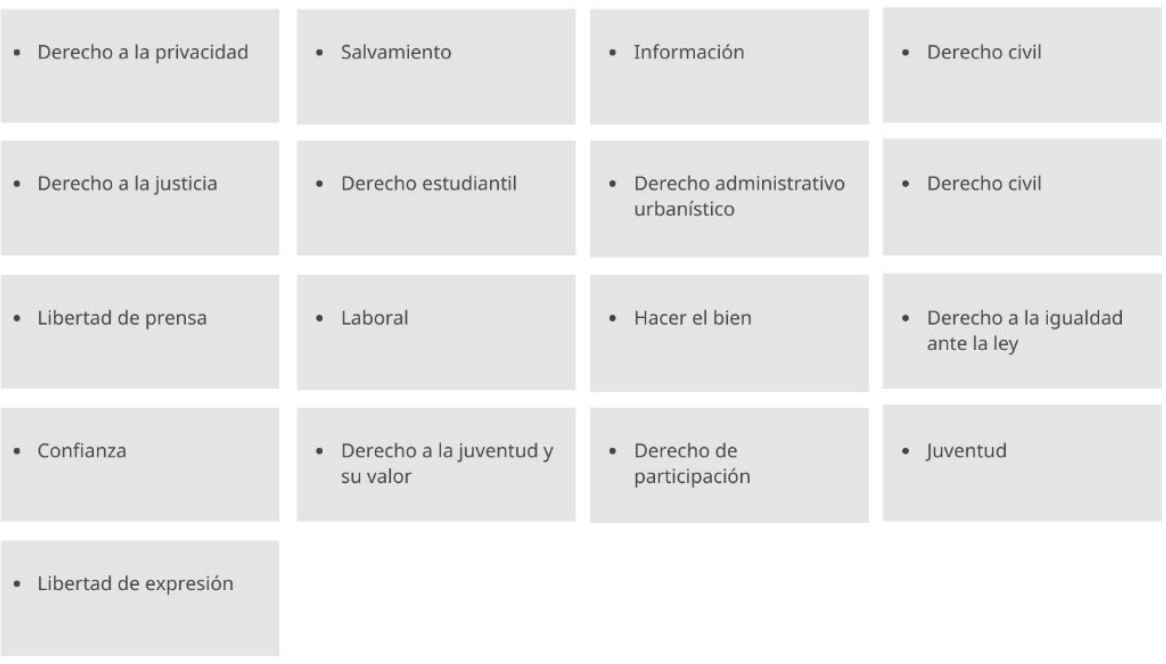

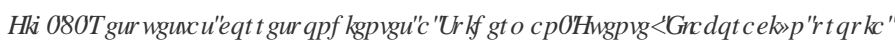

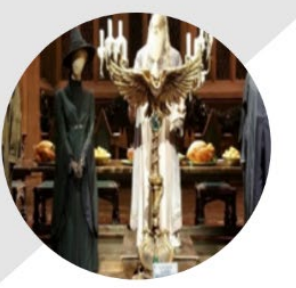

\section{Harry Potter}
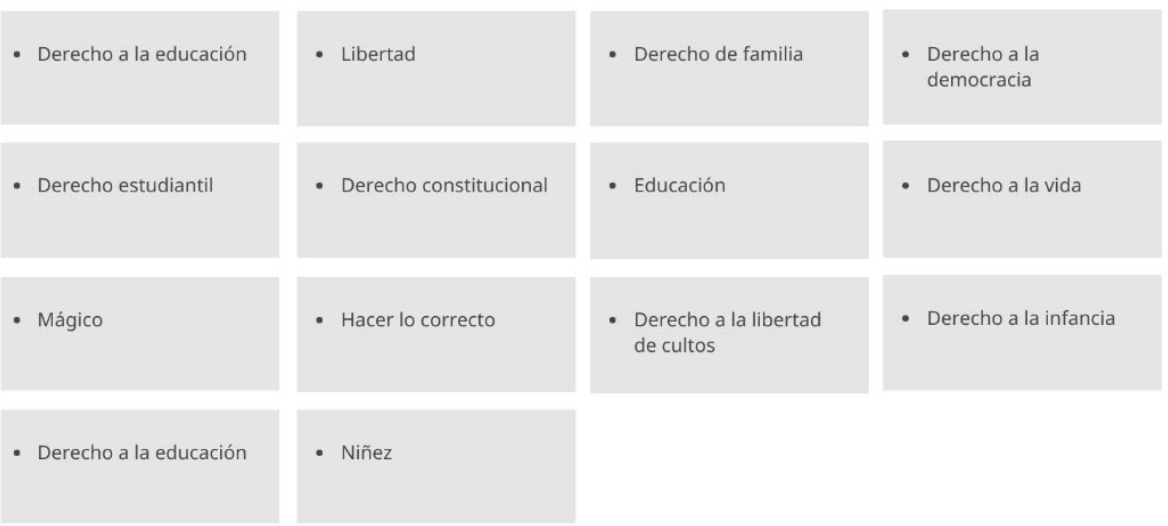

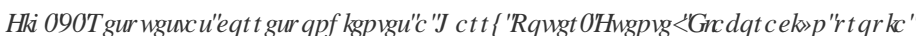

(c)) BY-NC-ND 2021, Universitat Politècnica de València

CRQJUHR], Q5 HGDपिएव 

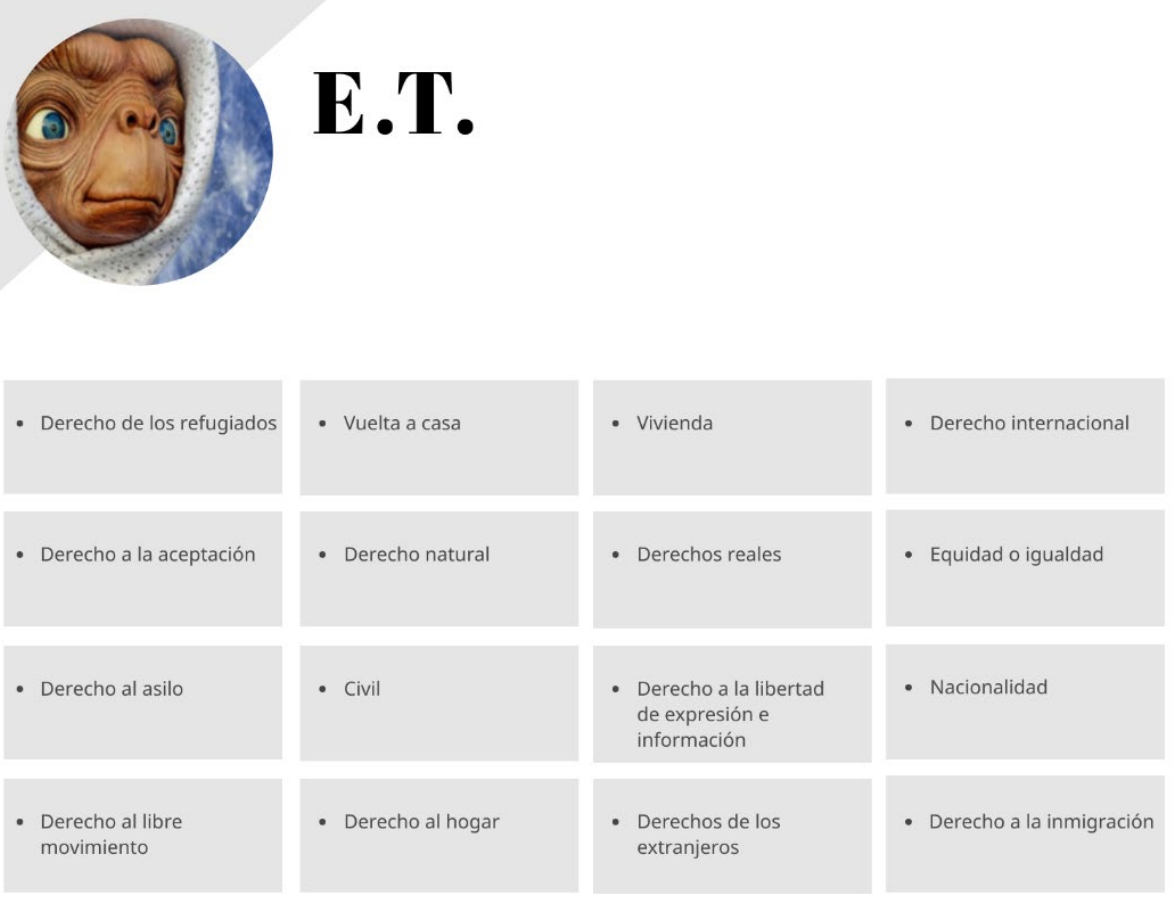

- Derecho a ser respetado

y no utilizado en

experimentos

biomédicos

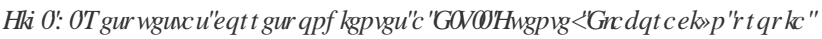

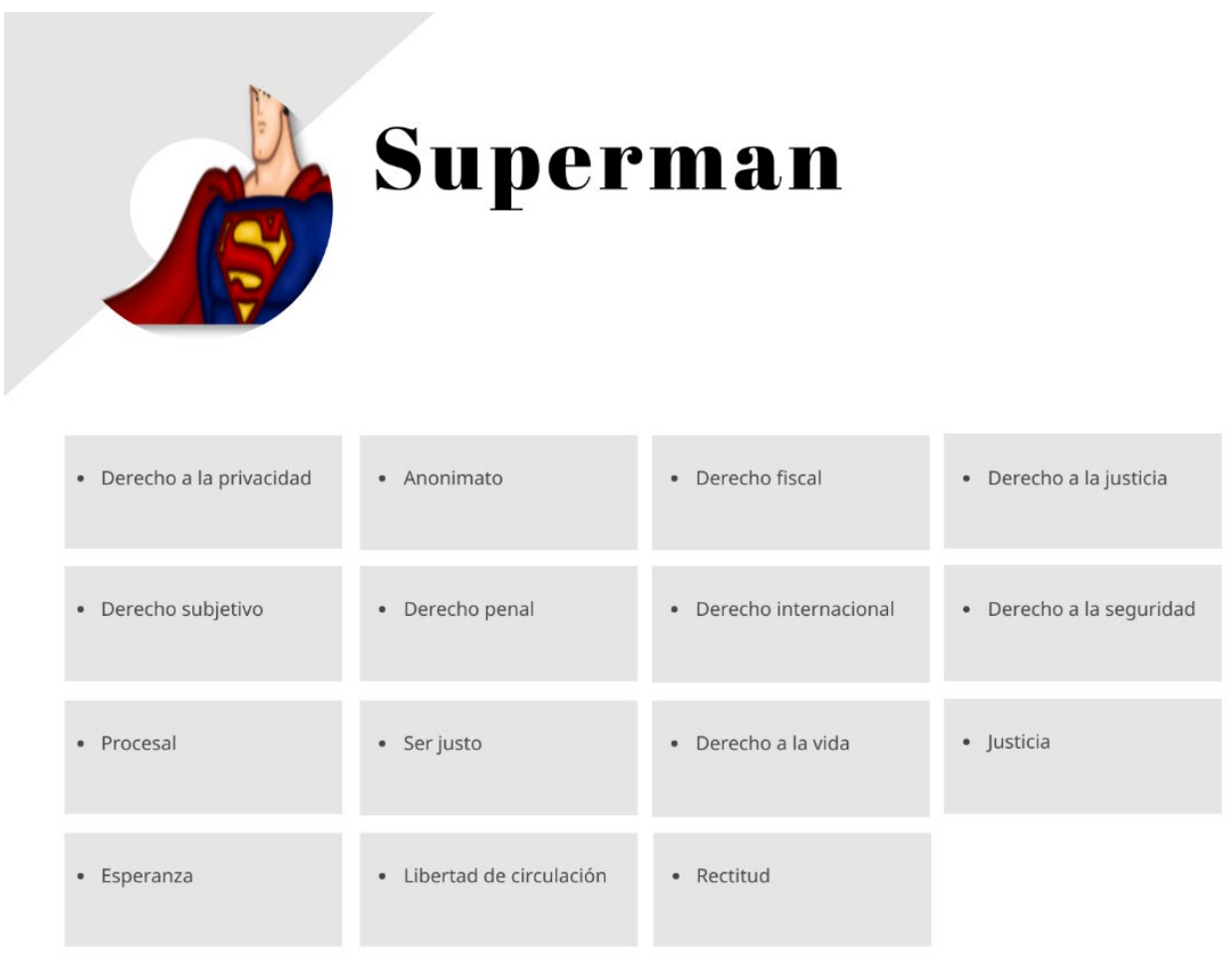

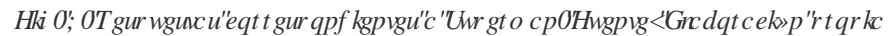

(cc) EY-NC-ND 2021, Universitat Politècnica de València

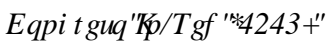




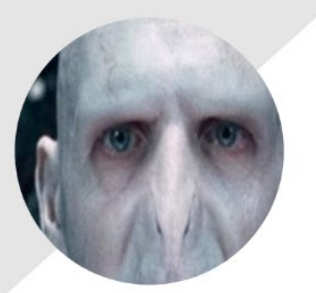

\section{Lord \\ Voldemort}
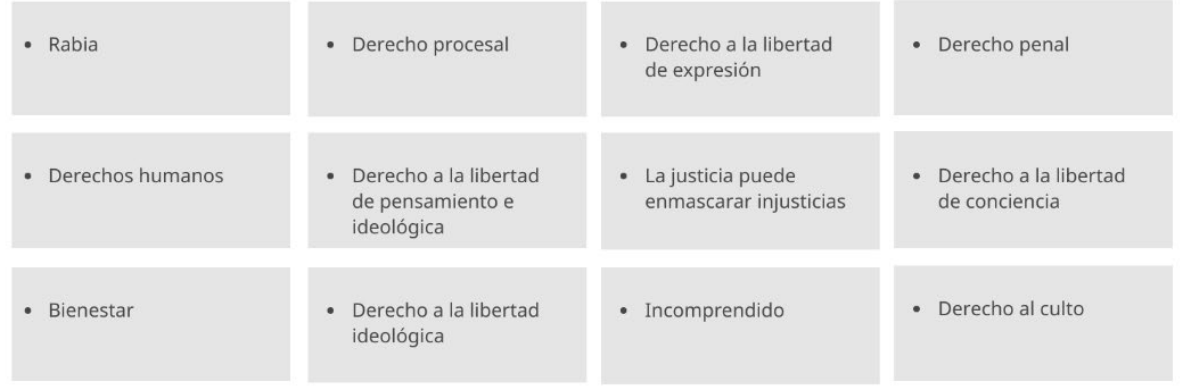

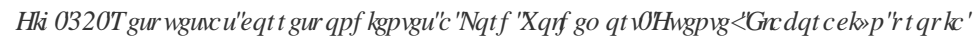

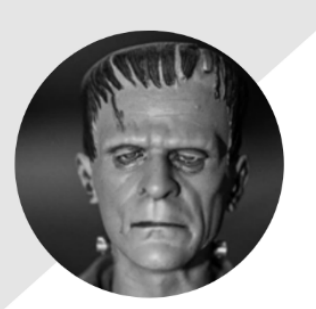

\section{Frankenstein}
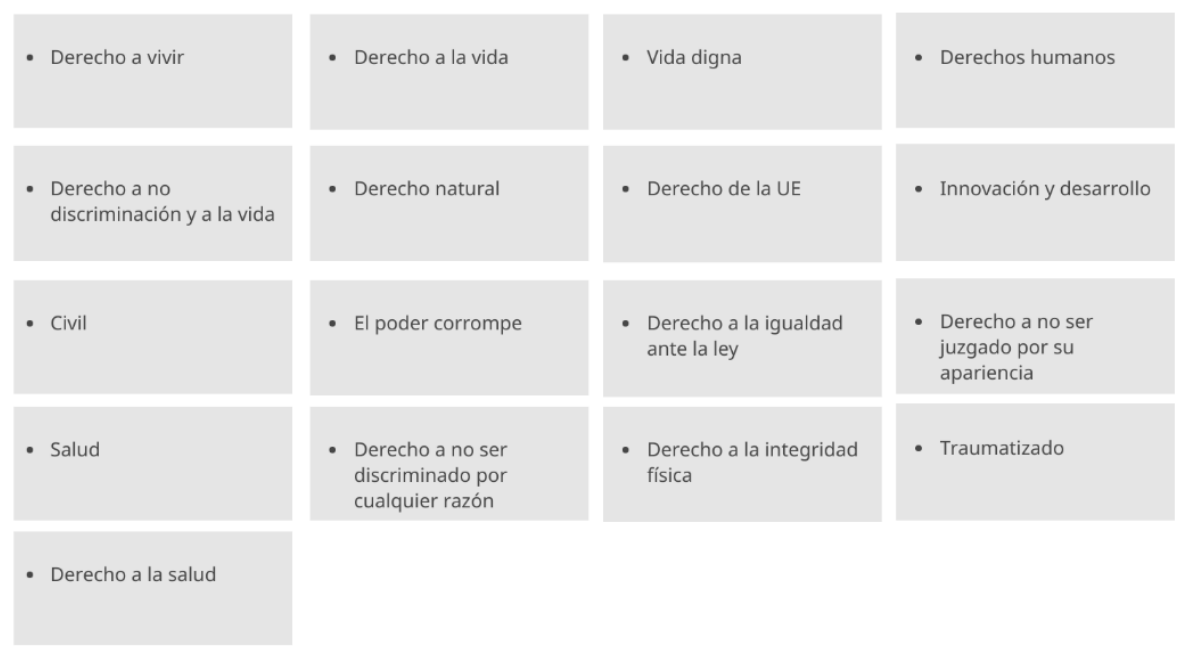

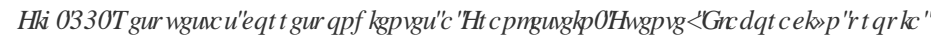

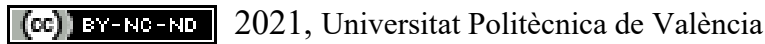

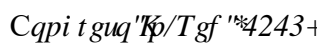




\section{Lobezno}
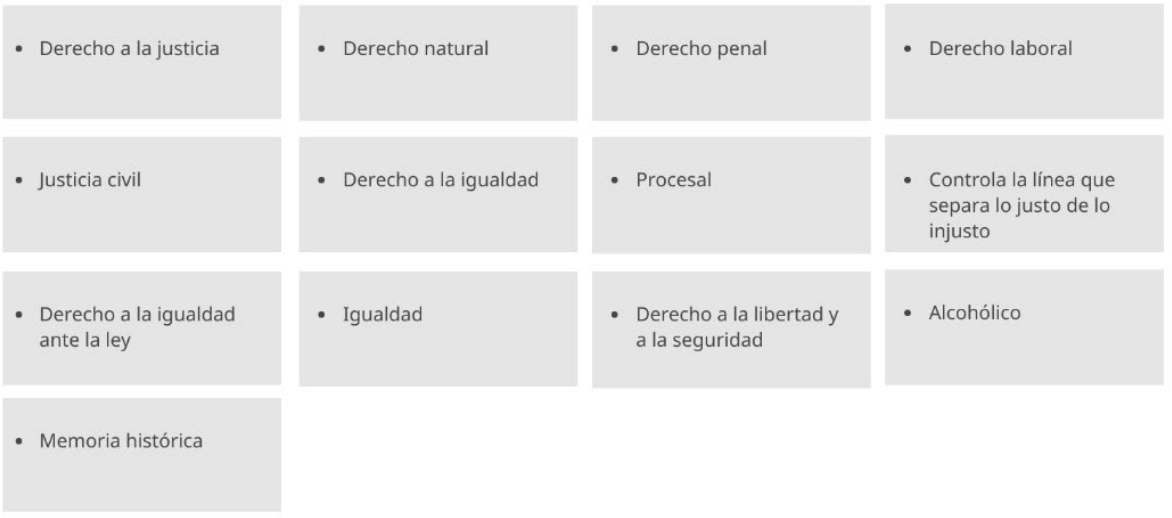

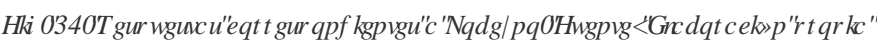

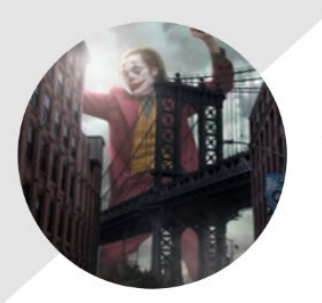

\section{EI Joker}
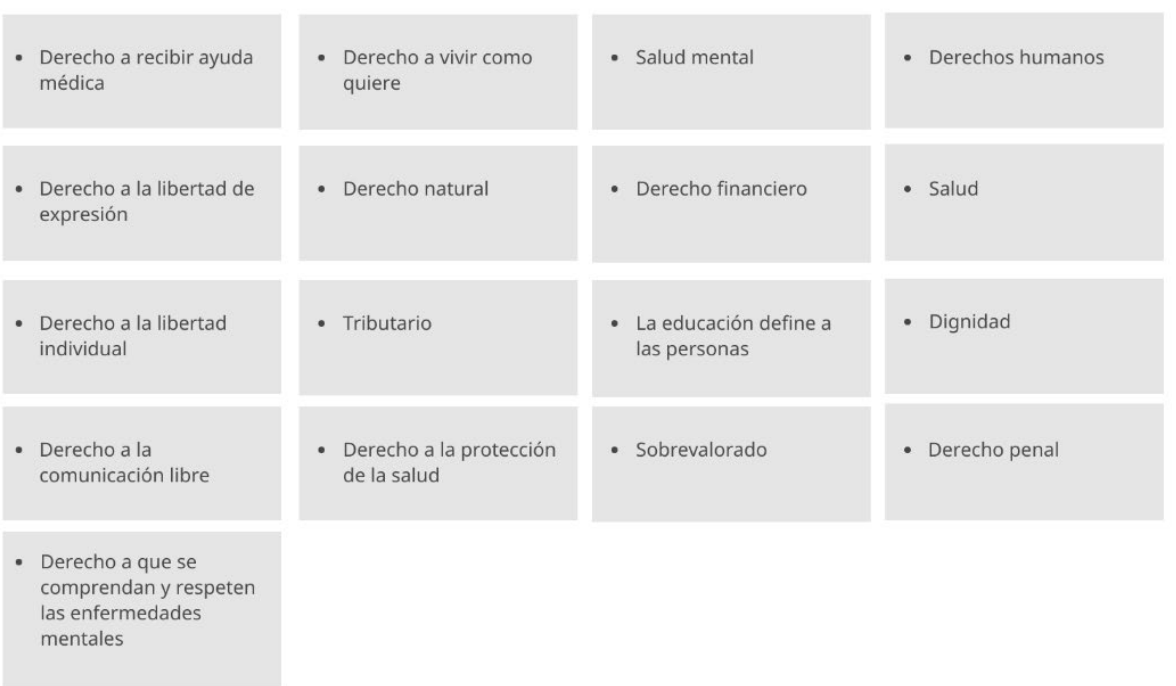

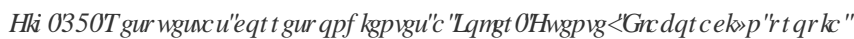

(cc) EY-NC-ND 2021, Universitat Politècnica de València

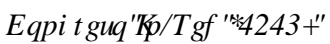




\section{Conclusiones}

Las principales conclusiones que hemos extraído de la innovación presentada han sido las siguientes:

1. Al alumnado le ha resultado una experiencia interesante ya que se trataba de superhéroes, y eran conocidos por todos. Se produce también una mayor dinamización en la asignatura, ya que el alumnado muestra su interés en participar, e incluso en las respuestas facilitadas las completa con la inclusión de preceptos jurídicos, por ejemplo, del ámbito constitucional en relación con los derechos fundamentales. Se cumple, por tanto, con los objetivos, principalmente, en lo relativo a la asociación de los personajes de ciencia ficción con el derecho que le relacionan.

2. Conocimiento por parte del alumnado de todos los superhéroes incluidos en la encuesta, por lo que ha facilitado la comprensión e identificación de los aspectos más relevantes de los personajes. Mejora su aprendizaje y su capacidad de comprensión de los conceptos jurídicos, ya que los asocia al superhéroe.

3. Alta capacidad de identificar los derechos asociados a los personajes de ciencia ficción. El alumnado identifica al superhéroe y al antihéroe en la lucha del bien y del mal, así como ha logrado identificar a los sujetos vulnerables, bien por sufrir una patología médica, bien por encontrarse en una situación de desamparo y vulnerabilidad de sus derechos.

4. El análisis de los resultados se ha realizado a través de Google Forms, en el que se ha vinculado el personaje de ciencia ficción con el derecho asociado, realizando un cómputo de respuestas en los derechos similares que han sido asociados.

4. La encuesta realizada nos ha servido para observar el nivel del alumnado respecto del conocimiento y asociación de conceptos jurídicos a través de los superhéroes. Esta gamificación ha supuesto un aliciente al alumnado y un instrumento de ayuda al profesorado para una nivelación de conocimientos.

5. Sirve como introducción para la asociación de conceptos a la actividad de resolución del caso práctico basado en la ciencia ficción, ya que le permite recordar los derechos asociados a los personajes, y una mayor ubicación conceptual de las ramas jurídicas: derecho civil, procesal, mercantil, financiero, constitucional.

\section{Agradecimientos}

Trabajo realizado en el marco del Proyecto de Innovación y Mejora Educativa (PIMEs) "Gamificación y TICs: diseño de actividades audiovisuales basadas en la ciencia ficción para la dinamización docente en un entorno presencial, semipresencial y virtual", presentado en el marco de la convocatoria de Proyectos de Innovación y Mejora Educativa (PIMEs) llevada a cabo en la Universitat Politècnica de València para el curso 2020-2022 obteniendo resolución favorable de la Comisión de Evaluación y Seguimiento de Proyectos de Innovación y Convergencia (CESPIC) en su sesión de septiembre de 2020 y concedido por el Vicerrectorado de Estudios y Convergencia Europea de la Universitat Politècnica de València. Años 20202022. Investigadora principal: Francisca Ramón Fernández. Investigadores: Vicente Cabedo Mallol, María Emilia Casar Furió, Vicent Giménez Chornet, Cristina Lull Noguera y Juan Vicente Oltra Gutiérrez, Amparo Soriano Soto, Laura Osete Cortina, Fernando Hernández Guijarro y Pilar Bosch Roig, y en el marco del Proyecto I+D+i «Retos investigación» del Programa estatal de I+D+i orientado a los Retos de la Sociedad del Ministerio de Ciencia, Innovación y Universidades: RTI2018-097354-B-100. «Contratos, transparencia y protección de datos en el mercado digital» (2019-2022). Investigadores Principales: Dr. Javier Plaza Penadés, Catedrático de Derecho Civil, y Dra. Luz M. Martínez Velencoso, Catedrática de Derecho Civil. Universitat de València-Estudi General. 


\section{Referencias}

Alerm GonzÁlez, A. y GonZÁlez Pérez, U. (2019). "Propuesta del cine de ciencia ficción para educar en Bioética”, 3 HURQDI [EIRpUAD, vol. 23, núm. 1, pp. 14-33.

$<$ https://personaybioetica.unisabana.edu.co/index.php/personaybioetica/article/view/8445/pdf $>$ [Consulta: 20 de enero de 2021].

CORCHUElo RodRíGUEZ, C. A. (2018). “Gamificación en educación superior: experiencia innovadora para motivar estudiantes y dinamizar contenidos en el aula", ( GXUFI5 HMLWD( OFWU QFDDHD HFQRQR TDD ( GXFDUYD, núm. 63, pp. 29-41.

$<$ https://doi.org/10.21556/edutec.2018.63.927> [Consulta: 10 de enero de 2021].

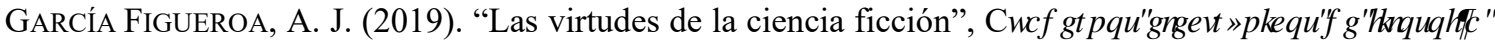
GHDCHHFRI núm. 40, pp. 265-291.

$<$ https://ojs.uv.es/index.php/CEFD/article/view/13885/pdf> [Consulta: 20 de enero de 2021].

García Martínez, J. L. y Brox Santiago, Ó. (2010). "Ciencia ficción, bioética. Desafíos en bioética desde el cine de David Cronenberg", en \%RpUFDHQ( XURSDI Andrés, J. R. (coord.), Instituto de Estudios Europeos y Derechos Humanos, Universidad Pontificia de Salamanca.

HERRERO, F. (2002). “Ciencia ficción y derecho”, / HपQRVDIV DUHLWD, núm. 30, pp. 44-45.

Martínez de Pisón CAvero, J. M". (2017). "Yo, Robot. De la biología a la singularidad. ¿Nuevas

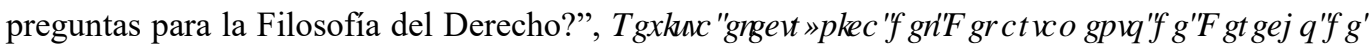
(D)8 QIYHUGDGGHV D5 IRND, núm. 15, pp. 57-73.

$<$ https://www.researchgate.net/profile/Jose_Cavero2/publication/325735471_Yo_Robot_de_la_biologia_ a_la_singularidad_Nuevas_preguntas_para_la_Filosofia_del_Derecho/links/5b20d6e20f7e9b0e3 73f2374/Yo-Robot-de-la-biologia-a-la-singularidad-Nuevas-preguntas-para-la-Filosofia-delDerecho.pdf $>$ [Consulta: 20 de enero de 2021].

Moreno Fuentes, E., Perales molada, R. Ma. e Hidalgo Navarrete, J. (2019). "Estudio cualitativo sobre el uso de la gamificación en Educación Superior para promover la motivación en el

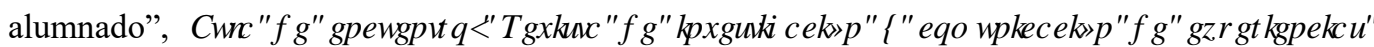
HGXFDUXDV, vol. 21, núm. 2, pp. 5-26.

$<$ https://revistaselectronicas.ujaen.es/index.php/ADE/article/view/5117/4645> [Consulta: 14 de enero de 2021].

OLTRA GUTIÉRREZ, J. V. et al. (2016). "Uso de fragmentos de películas y series como herramientas de innovación docente. Una experiencia con alumnos de informática", en ,15(' ए,, $\& \& R Q U H R]$ 1 DFIRQDOCH, QQRYDFIy Q( GXFDUYDI I' RFHQFDHQ5 HG Universitat Politècnica de València.

PÉREZ-LÓPEZ, I. y Rivera GARCíA, E. (2017). "Formar docentes, formar personas: análisis de los aprendizajes logrados por estudiantes universitarios desde una experiencia de gamificación", 6IJQR] [3 HQMP IHQUR, vol. 36, núm. 70, pp. 112-129.

$<$ https://www.edutec.es/revista/index.php/edutec-e/article/view/1247/665 $>$ [Consulta: 15 de enero de 2021].

PRENSKY, M. (2002)."«The motivation of gameplay: The real twenty-first century learning revolution”, 2 Q $U K H+R U R Q$ vol. 10, núm. 1, pp. 5-11. 
$<$ https://doi.org/10.1108/10748120210431349> [Consulta: 20 de enero de 2021].

PRIETO ANDREU, J. M. (2020). “Una revisión sistemática sobre gamificación, motivación y aprendizaje en universitarios", Teoría de la educación, vol. 32, núm. 1, pp. 73-199.

$<$ https://revistas.usal.es/index.php/1130-3743/article/view/teri.20625/21290> [Consulta: 20 de enero de 2021].

RAMÓN FERNÁNDEZ, F. (2019). "La enseñanza del derecho civil a través del elemento audiovisual en titulaciones no jurídicas: una innovación docente en la Universitat Politècnica de València", 5HILWD( GXFDFlyQI Q' HHFKR, núm. 20, pp. 1-23.

$<$ http://revistes.ub.edu/index.php/RED/article/view/29704/30032> [Consulta: 20 de enero de 2021].

RAMÓN FERNÁNDEZ, F. et al. (2016). \&DVRUSU FWFRVMUUGFRVEDWDRVHQQUHHWGHDQPP DFIyQ Francisca Ramón Fernández (coord.). Valencia: Tirant lo Blanch.

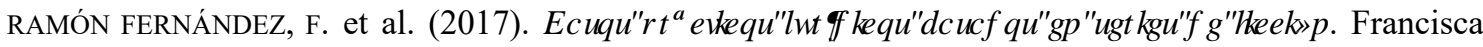
Ramón Fernández (coord.). Valencia: Tirant lo Blanch.

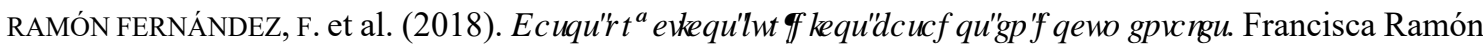
Fernández (coord.). Valencia: Tirant lo Blanch.

RAMÓN FERNÁNDEZ, F. et al. (2019a). \&DVRUSU FUFRVUXUGGFRVEDWDRVHQHDKXP RU Francisca Ramón Fernández (coord.). Valencia: Tirant lo Blanch.

RAMÓN FERNÁNDEZ, F. et al. (2016a). "Diseño de casos prácticos jurídicos basados en series de animación. Una innovación docente con soporte audiovisual”, en ' HHFKR]। 7, \& [ [ QWP DV LQQRYDFIRQHV] GRFQUN, Coordinadores Ana María Delgado García e Ignacio Beltrán de Heredia Ruiz. Barcelona: Huygens editorial, pp. 123-132.

RAMÓN FERNÁNDEZ, F. et al. (2016b). "Utilización de las series de animación como recurso didáctico en la elaboración de casos. Análisis de una experiencia en la innovación docente en la Universitat

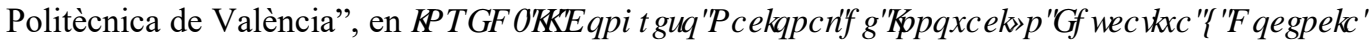
HQ5 HG Universitat Politècnica de València.

RAMÓN FERNÁNDEZ, F.et al. (2019c). "Diseño de materiales docentes basados en recursos audiovisuales de humor para la mejora del aprendizaje y su evaluación en ciencias sociales y jurídicas. Análisis del diseño de la actividad sobre las casas Cueva y «Los Picapiedra» en / DQRFHQFDGHO HHFRRHQ QDURFHCDEGJLMOB Barcelona: Huygens Editorial.

Ramón Fernández, F., Lull Noguera, C., Soriano Soto, Ma. D. (2019). “Actividades docentes del PYME. Diseño de materiales docentes para la mejora del aprendizaje y su evaluación en ciencias

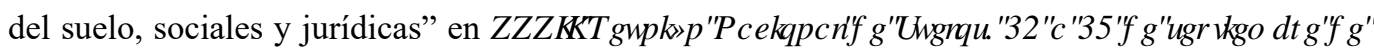
૧૯० RENS. Sevilla: Sociedad Española de la Ciencia del Suelo.

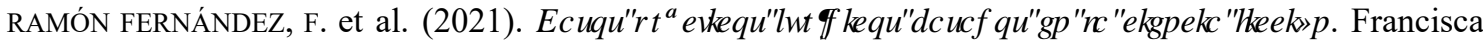
Ramón Fernández (coord.). Valencia: Tirant lo Blanch. 